\title{
Antiretroviral therapy and cardiovascular risk
}

\author{
E Ridha, E Devitt, M Boffito, F Boag \\ Department of HIV Medicine, Chelsea and Westminster Hospital, London, UK \\ Correspondence to E Ridha, essra_ridha@hotmail.com
}

\begin{abstract}
Summary
The increase in the risk of myocardial infarction can be explained by antiretroviral-induced changes in conventional cardiovascular risk factors. Individual drugs within a drug class vary in their propensity to cause metabolic disturbances, and therefore, further studies are needed to determine the contribution of each drug to cardiovascular risk. A careful stratification of the cardiovascular risk and cardiovascular monitoring of HIV-infected individuals should be performed at baseline and at regular intervals during follow-up for this chronic medical condition. Standard primary and secondary prevention measures should be instituted in accordance with British Guidelines (Heart association, National Institute for Health and Clinical Excellence, etc for lipids, smoking and hypertension) Consideration of cardiovascular risk and drug-drug interactions when prescribing is crucial in the safe and optimum management of these patients. Careful communication between all care providers will help to avoid adverse outcomes.
\end{abstract}

\section{BACKGROUND}

With the advent of highly active antiretroviral therapy (HAART) for HIV infection, the morbidity and mortality associated with AIDS defining conditions has been dramatically reduced. ${ }^{1}$ As a result, HIV-infected individuals are living longer and developing common medical conditions in line with the general population. ${ }^{1}$ In addition, metabolic issues such as abnormalities of lipid metabolism, insulin resistance, hyperglycaemia and lipodystrophy are becoming more prevalent. ${ }^{1}$ The incidence of cardiovascular disease including hypertension and coronary artery disease is on the increase in the HIV-positive population. ${ }^{1}$ The traditional risk factors still apply in this sub-population; however, HIV itself and its effective treatment with HAART contribute to the metabolic and cardiovascular risk profile of an individual. ${ }^{1} 2$

HAART has been implicated in the aggravation of coronary artery disease and myocardial infarction, in particular the class-specific metabolic effects of protease inhibitors is thought to have a significant deleterious effect on progression of disease and therefore an increased rate of myocardial infarction. ${ }^{2}$

Cardiovascular risk and the rate of myocardial infarction in patients infected with HIV have been assessed in 13 drugs in the Data Collection on Adverse Events of AntiHIV Drugs (DAD) study, which suggested that protease inhibitors indinavir, lopinavir-ritonavir and nucleoside reverse transcriptase inhibitors didanosine and abacavir were associated with significantly increased risk. ${ }^{3}$

The pathogenic mechanism by which these drugs contribute to cardiovascular events remains unclear but several preliminary studies have proposed that abacavir may promote systemic inflammation and platelet adhesion as evidenced by increased inflammatory markers in these patients. This subsequently contributes to atherosclerotic plaque instability and rupture. ${ }^{4}$

It is essential to consider an individual's cardiovascular risk when prescribing HAART in order to minimise any adverse long-term effects of therapy. However, often it is difficult to modify lifesaving HAART regimens in more complex treatment-experienced patients with drug resistant HIV as options can be limited. It is this particular subgroup of older, complex treatment-experienced patients who require meticulous prescribing with careful attention to all potential interactions due to polypharmacy.

Primary and secondary prevention strategies to reduce the risk of vascular events by reducing the risk factors are increasingly difficult due to drug interactions between HAART and several classes of drugs, including ACE inhibitors, calcium channel blockers, $\beta$ blockers, statins and diuretics.

Here we describe a challenging case to illustrate some of these issues.

\section{CASE PRESENTATION}

A 43-year-old male, who was initially diagnosed HIV positive in 1989, received several drug regimens before the advent of combination HAART in the late 1990s. As a result of this, he developed triple class resistant HIV. He was also found to be hepatitis B co-infected in 2000. Since 2007 he has been maintained on salvage therapy with tenofovir $245 \mathrm{mg}$ once daily, emtricitabine $200 \mathrm{mg}$ once daily, darunavir $800 \mathrm{mg}$ once daily, ritonavir $100 \mathrm{mg}$ once daily, maraviroc $150 \mathrm{mg}$ twice daily and raltegravir 400 mg twice daily. His viral load remains undetectable with a CD 4 count of 243 on this regimen.

In 2002, he presented with high grade non-Hodgkin's lymphoma (stage IVB) with renal involvement for which he received cyclophosphamide, doxorubicin and etoposide (regimen) chemotherapy given monthly for six cycles with granulocyte colony-stimulating factor support and intrathecal chemoprophylaxis. He has been in remission since 2003.

In 2008 , he was noted to be hypertensive when he presented to hospital with severe headache and blood pressure ranging between 150-160 mm $\mathrm{Hg}$ systolic and 100-110 $\mathrm{mm} \mathrm{Hg}$ diastolic. His antiretroviral (HRV) regimen at this time included tenofovir $245 \mathrm{mg}$ once daily, emtricitabine 
$200 \mathrm{mg}$ once daily, darunavir $800 \mathrm{mg}$ once daily, ritonavir $100 \mathrm{mg}$ once daily, maraviroc $150 \mathrm{mg}$ twice daily and raltegravir $400 \mathrm{mg}$ twice daily. He was commenced on low dose perindopril, which was subsequently changed to bendroflumethazide due to impaired renal function (urea 8.1, creatinine 136, epidermal growth factor receptor (eGFR) 49) and the likelihood of interactions between protease inhibitors (ritonavir, darunavir) and perindopril, which would act to increase the level and toxicity of the drug. His lipids were elevated at this time and he commenced atorvastatin (total cholesterol measurement of 6.2).

Investigations for causes of secondary hypertension and impaired renal function were performed. Imaging revealed large kidneys with lobulation and multiple deposits unchanged from 2002 when his renal function had been normal (urea 4.2, creatinine 82, eGFR >90). An magnetic resonance angiography of his renal arteries revealed no abnormalities. All other investigations for secondary hypertension and impaired renal function were negative/ normal.

An echocardiogram revealed normal left ventricular cavity size and wall thickness with good systolic function.

In 2008, he was also diagnosed with migraine and treatment with propranolol $10 \mathrm{mg}$ once daily and intermittent sumatriptan was commenced, which effectively controlled his symptoms.

His hypertension remained uncontrolled despite treatment with bendroflumethazide and propranolol. He was commenced on low dose ramipril in a specialist renal clinic that recommended slow uptitration of the ACE inhibitor in combination with the thiazide diuretic and close monitoring of renal function with the aim of achieving a blood pressure consistently lower than 130/80 $\mathrm{mm} \mathrm{Hg}$ to reduce his cardiovascular risk and prevent further decline in renal function. Further options to achieve such a blood pressure include a calcium antagonist and $\alpha$ blocker as recommended by the renal physicians.

However, the potential for harmful interaction between calcium channel blockers and ritonavir make this class of drug an unattractive option for treatment of hypertension in this situation. Propranolol was also given with caution when combined with a boosted protease inhibitor due to the potential for toxicity in CYP2D6 slow metabolisers. As the patient had experienced no adverse effects on the propranolol, it was decided that he should continue with the current dose of propranolol (10 mg once daily) and not increase it further.

Follow-up and close monitoring of his renal function demonstrated worsening of renal function upon uptitration of the ACE inhibitor. Furthermore, the patients' blood pressure remained elevated at 140-150/90-100 mm Hg despite treatment with bendroflumethazide, ramipril and propranolol.

At this stage, it was decided that the HRV regime should be altered in view of the deterioration in renal function. The dose of the tenofovir was reduced to $245 \mathrm{mg}$ on alternate days. The dose of the darunavir/ritonavir was changed to 800/100 once daily with the aim of reducing the daily ritonavir dose and decreasing the likelihood for interactions should further antihypertensives be required/ added at a later stage. More regular monitoring of viral load was initiated at this point as the doses of ARVs had been altered. His HIV viral load remained undetectable.
Despite the changes made, his hypertension remained uncontrolled and diltiazem $60 \mathrm{mg}$ once daily was commenced.

He was referred for a cardiology opinion and the dose of diltiazem was doubled to $120 \mathrm{mg}$ and 24-h blood pressure monitoring was arranged, which revealed a mean systolic blood pressure of $150 \mathrm{~mm} \mathrm{Hg}$ and diastolic pressure of 90 $\mathrm{mm} \mathrm{Hg}$.

A combination of bendroflumethazide, ramipril, propranolol and diltiazem controlled his blood pressure effectively at this stage achieving pressures consistently lower than 130/80 $\mathrm{mm} \mathrm{Hg}$, following which he presented to hospital with symptomatic hypotension. The diuretic and diltiazem were discontinued and he now remains normotensive on irbesartan $150 \mathrm{mg}$ and propranolol $40 \mathrm{mg}$.

\section{DISCUSSION}

Since the introduction of HAART, there have been dramatic reductions in the prevalence of HIV-associated cardiomyopathy secondary to myocarditis and the prevalence of cardiac involvement of AIDS-associated malignancies. ${ }^{6}$ However, excess cardiovascular risk in HIV-infected individuals has been a growing concern in which traditional risk factors such as smoking and ageing in combination with HIV-induced immune activation and endothelial dysfunction and therapy-associated dyslipidaemia, hypertension and insulin resistance aggravate the risk of cardiovascular disease and increase the rate of MI. ${ }^{4}$

HIV-induced immune activation and the effective treatment of HIV with HAART contributes to the metabolic and cardiovascular risk profile of an individual ${ }^{4}$ by promoting atheroma formation, plaque instability, hypercoagulation and systemic inflammation all of which accelerate atherosclerotic processes. ${ }^{4}$

The HRV therapy regimen is a modifiable risk factor, as there are class differences and within-class differences in the propensity to increase metabolic disturbance. Careful cardiovascular evaluation in the course of HIV disease can identify cardiac complications early enough to treat. All HIV-infected patients are candidates for HRV therapy and patients already on treatment should undergo an assessment that includes the evaluation of the cardiovascular risk. The Framingham calculator is a tool used to estimate risk of myocardial infarction and death from coronary artery disease within the next 10 years. This estimation is based on a score developed in the Framingham heart study.

\section{Learning points}

- The increase in the risk of myocardial infarction can be explained by HRV-induced changes in conventional cardiovascular risk factors.

- A careful stratification of the cardiovascular risk and cardiovascular monitoring of HIV-infected individuals should be performed at baseline and at regular intervals during follow-up for this chronic medical condition. ${ }^{8}$

- Standard primary and secondary prevention measures should be instituted in accordance with British Guidelines. Consideration of cardiovascular risk and drug-drug interactions when prescribing is crucial in the safe and optimum management of these patients. 


\section{BMJ Case Reports}

As highlighted by our report, physicians treating patients receiving HRV therapy should be especially aware of the potential for harmful interactions between anti-HIV drugs and anti-hypertensive and lipid-lowering drugs.

Competing interests None.

Patient consent Obtained.

\section{REFERENCES}

1. Mary-Krause M, Cotte L, Simon A, et al.; Clinical Epidemiology Group from the French Hospital Database. Increased risk of myocardial infarction with duration of protease inhibitor therapy in HIV-infected men. AIDS 2003;17:2479-86.

2. Friis-Møller N, Reiss P, Sabin CA, et al.; DAD Study Group. Class of antiretroviral drugs and the risk of myocardial infarction. N Engl J Med 2007:356:1723-35
3. Worm SW, Sabin C, Weber R, et al. Risk of myocardial infarction in patients with HIV infection exposed to specific individual antiretroviral drugs from the 3 major drug classes: the data collection on adverse events of anti-HIV drugs (D:A:D) study. J Infect Dis 2010;201:318-30.

4. Behrens GM, Reiss P. Abacavir and cardiovascular risk. Curr Opin Infect Dis 2010;23:9-14

5. Hürlimann D, Weber $\mathrm{R}$, Enseleit $\mathrm{F}$, et al. [HIV infection, antiretroviral therapy, and endothelium]. Herz 2005;30:472-80.

6. Barbaro G. Reviewing the cardiovascular complications of HIV infection after the introduction of highly active antiretroviral therapy. Curr Drug Targets Cardiovasc Haematol Disord 2005;5:337-43.

7. Bergersen BM. Cardiovascular risk in patients with HIV Infection: impact of antiretroviral therapy. Drugs 2006:66:1971-87.

8. Law MG, Friis-Møller N, El-Sadr WM, et al.; D:A:D Study Group. The use of the Framingham equation to predict myocardial infarctions in HIV-infected patients: comparison with observed events in the D:A:D Study. HIV Med 2006; 7:218-30.

This pdf has been created automatically from the final edited text and images.

Copyright 2011 BMJ Publishing Group. All rights reserved. For permission to reuse any of this content visit

http://group.bmj.com/group/rights-licensing/permissions.

BMJ Case Report Fellows may re-use this article for personal use and teaching without any further permission.

Please cite this article as follows (you will need to access the article online to obtain the date of publication).

Ridha E, Devitt E, Boffito M, Boag F. Antiretroviral therapy and cardiovascular risk. BMJ Case Reports 2011;10.1136/bcr.10.2010.3429, date of publication

Become a Fellow of BMJ Case Reports today and you can:

- Submit as many cases as you like

- Enjoy fast sympathetic peer review and rapid publication of accepted articles

- Access all the published articles

- Re-use any of the published material for personal use and teaching without further permission

For information on Institutional Fellowships contact consortiasales@bmjgroup.com

Visit casereports.bmj.com for more articles like this and to become a Fellow 